\title{
Beef Meat Allergy in Cow's Milk Allergic Adults
}

\author{
Maged Refaat ${ }^{1 *}$, Amany Kamal ${ }^{1}$, Mohamed Fares ${ }^{1}$, Eman Ossman ${ }^{1}$, Mohamed Attia $^{1}$, Alsayed Elokda ${ }^{2}$ \\ ${ }^{1}$ Department of Internal Medicine, Allergy and Clinical immunology, Ain Shams University Hospital, Cairo, Egypt; ${ }^{2}$ Department of \\ Community, Environmental and Occupational Medicine, Faculty of Medicine, Ain Shams University, Cairo, Egypt. \\ Email: "dr_maged_refaat@hotmail.com.
}

Received July 10 $0^{\text {th }}$, 2011; revised August $8^{\text {th }}$, 2011; accepted August $15^{\text {th }}$, 2011.

\begin{abstract}
Milk has been recognized as a leading cause of food allergy in children; however, studies on cow's milk allergy (CMA) in adults are scanty. The known cross-reactivity of bovine serum albumin and bovine $\gamma$-globulin which are present in both milk and beef is recognized, so that, patients with allergy to cow's milk are often instructed to avoid beef. Our Objective was to determine the prevalence of allergy to beef meat in adult patients with allergy to cow's milk. Thirty adults with CMA were included in the study. The diagnosis of cow's milk and beef allergy was based on a thorough history concerning type of allergic symptoms and its link to the ingestion of milk and beef supported by skin prick test (SPT) with fresh milk and raw beef extracts and by determination of their serum specific IgE by enzyme linked immuosorbant assay (ELISA). Finally, cross reactivity test between milk and meat was performed. Three of 30 patients (10\%) evaluated for CMA were found to have symptomatic sensitivity to beef, 2 gave urticarial symptoms and one gave gastrointestinal symptoms. Six patients (20\%) had a positive SPT response to beef. On the other hand, beef specific IgE were positive in 18 CMA patients (60\%). Concordance of positive specific IgE and SPT to meat was found in only 5 patients (17\%). Mean cross reactivity between milk and beef was $31.6 \% \pm 13.1 \%$ among studied patients. Patients allergic to milk protein are not necessarily to be allergic to beef, so, elimination of beef from the diet of adults with CMA should not be done except after investigations so as not to lose its nutritional value without benefit.
\end{abstract}

Keywords: Beef, Meat, Milk, Allergy, Adults

\section{Introduction}

Milk has been recognized for many years as one of the leading causes of food allergies. Epidemiological data indicate that the prevalence of cow's milk allergy is approximately $1 \%$ to $2 \%$ [1]. Meat allergy is also relatively frequent, and beef proteins are among the most common allergens involved in food hypersensitivities. The major allergens of meat are serum albumins and immunoglobulins, but there are a few reports of allergies to muscle proteins (actin, myosin and tropomyosin) [2].

The known allergenic cross-reactivity of bovine serum albumin and bovine $\gamma$-globulin, which are present in both milk and beef, has been noted as a cause for concern; a higher prevalence of beef allergy might be expected among children allergic to milk [3]. Furthermore, cooking or other processing methods do not necessarily eliminate the allergenic epitopes from milk and meat products [4].

Studies on cow's milk allergy in adults are scarce [5]. Patients with allergy to cow's milk are often instructed to avoid beef in their diet. The removal of beef and milk from the diet of beef-allergic patients may pose nutritional problems, as milk, dairy products, and meat represent an important source of proteins in diet.

However, there is no scientific basis for the elimination of beef from the diet of patients with cow's milk allergy. Epidemiologic data have not addressed the prevalence of beef allergy relative to cow's milk allergy, or the possibility of an overlap of these two conditions [3].

Some authors have even observed that heat treatment reduced the allergenicity of beef, adding that since meat was predominantly consumed in cooked form, the partial heat labiality of major beef allergens contributed to the general low prevalence of beef allergy [6,7]. Others added that beef did not need to be excluded form the diet of patients allergic to cow's milk as long as it was eaten cooked [8].

Diagnosis of cow's milk allergy and meat allergy is primarily based on skin prick tests and measurement of cow's milk or beef-specific IgE antibodies. Adults with CMA display a stronger SPT and IgE reactivity [5]. In selected patients, a standardized food challenge might be necessary to determine the diagnosis [1].

The aim of the study was to determine the prevalence of allergy to beef meat in adult patients with allergy to 
cow’s milk.

\section{Materials and Methods}

We included 30 cow's milk allergic adult's recruited form the outpatient's immunoallergy clinic of Ain Shams University hospital during the period of October 2010 to March 2011. The cross sectional study was approved by the review board of the allergy and clinical immunology department, Ain Shams University, Cairo.

\subsection{Diagnosis}

The diagnosis of cow's milk and beef allergy was based on a thorough case history supported by skin prick tests (SPT) and serum specific IgE for milk and beef. Oral challenges were not done in this work due to the severity of the reactions and/or the patient's refusal.

The SPT were performed with a commercially available raw beef extract and fresh milk, saline solution and histamine control. A positive SPT reaction was defined as a wheal $>3$ millimeters larger than the control. Serum specific IgE were determined by enzyme linked immunusorbant assay (ELISA) and was used for quantitative determination of IgE in human serum (G. enzyme diagnostics). A specific IgE level greater than $0.35 \mathrm{Ku} / \mathrm{L}$ was considered positive according to [9].

Finally, a cross reactivity test between milk and beef was performed.

\subsection{Procedure of Agar Gel Precipitation for Cross Reactivity}

Gel diffusion, using Outchterlony method was done.

\subsubsection{Gel Preparation}

Agarose powder $3 \mathrm{gm}$ was added to agar-agar powder 2 gm in $250 \mathrm{ml}$ distilled water, then sterilized in the autoclave and poured in the Petri dish with thickness not exceeding $2 \mathrm{~mm}$ in diameter. Multiple wells were done in the plate.

\subsubsection{Cross Reactivity Test}

\section{1) Reactivity to milk}

The gel was cooked with milk antigen extract then, we put the serum of the patients each in a separate well on the plate. Incubation at $37^{\circ} \mathrm{C}$ for 48 hours was done. We read the diameter of the immunodiffusion (reaction between the milk antigen and its antibodies).

2) Reactivity to beef

Here, the gel was cooked with beef antigen extract.

3) Cross reactivity between milk and beef

For each patient the diameter of immunodiffusion of milk was divided by that of beef. The percentage obtained was considered the cross reactivity between milk and beef of this patient [10].

\section{Results and Discussion}

\subsection{Milk Allergic Patients}

Thirty adults were diagnosed to have milk allergy by relevant history, positive skin prick test for fresh milk and serum specific IgE. The patients' age range was 16 to 39 years with mean age $21.4 \pm 6.8$ years. 17 patients were males (56.6\%). Their disease duration was ranged from 0.25 to 15 years (mean $3.8 \pm 3.2$ years).

The symptoms were urticaria in 18 patients (60\%), gastrointestinal in 7 patients (23\%) and respiratory (nasobronchial) in 5 patients (17\%).

\subsection{Beef Allergic Patients}

Results concerning beef allergy (Table 1) demonstrated a relevant history to meat allergy was obtained in 3 patients (10\%), 2 gave urticarial symptoms and one gave gastrointestinal symptoms. 6 patients (20\%) gave positive SPT to meat allergen, 3 of them (10\%) were symptomatic to meat. 18 patients $(60 \%)$ had positive specific IgE to meat allergens (>0.35 KU/L).

There was no significant relation between skin prick test and specific IgE to meat $(\mathrm{P}>0.05)$ as in Table 2. Concordance of positive specific IgE and SPT to meat was found only in 5 patients (17\%).

\subsection{Cross Reactivity Test}

Among studied patients Cross reactivity test between milk and beef, it was ranged from $8 \%$ to $60 \%$. The mean percentage was $31.6 \% \pm 13.1 \%$.

\subsection{Diagnosis of Milk and Beef Allergy}

Our study included 30 adults who had milk allergy. Their diagnosis were based on a thorough history concerning type of allergic symptoms and its link to the ingestion of milk, positive SPT for fresh milk and serum specific IgE. Among cases with milk allergy, diagnosis of cases allergic to beef were based on a relevant history, SPT to raw beef extract and serum specific IgE. Oral challenges were not done in our work due to the severity of the reactions expected in adults [5], in addition to patient's refusal. CMA is of two types: IgE mediated and non IgE mediated allergy. According to Martorell study, IgE mediated allergy is the main type in CMA [8], where among 249 patients confirmed to have CMA by oral food challenge test, IgE mediated CMA was diagnosed in 234 patients (94\%) and non IgE mediated hypersensitivity to CMA in 15 patients (6\%).

\subsection{Allergic Symptoms}

In our study, 18 out of 30 adults with CMA (60\%) had skin allergy while 7 out of 30 (23\%) had gastrointestinal symptoms and 5 had respiratory symptoms (17\%). This 
Table 1. Relation between cow milk allergy versus beef allergy by symptoms, skin prick test and specific IgE.

\begin{tabular}{|c|c|c|}
\hline Diagnosis of beef allergy (BA) & Cases with CMA $(\mathrm{n}=30)$ & Percent \% \\
\hline \multicolumn{3}{|l|}{ 1. Symptoms } \\
\hline Negative & 27 & $90 \%$ \\
\hline Positive & 3 & $10 \%$ \\
\hline \multicolumn{3}{|l|}{ 2. Skin prick test } \\
\hline Negative & 24 & $80 \%$ \\
\hline Positive & 6 & $20 \%$ \\
\hline \multicolumn{3}{|l|}{ 3. Specific IgE } \\
\hline Negative $(<0.35 \mathrm{KU} / \mathrm{L})$ & 12 & $40 \%$ \\
\hline Positive (>0.35 KU/L) & 18 & $60 \%$ \\
\hline
\end{tabular}

CMA: cow milk allergy; BA: beef allergy.

Table 2. Relation between beef IgE versus skin prick test.

\begin{tabular}{cccc}
\hline & & SPT & \\
Beef IgE & Negative & Positive & P \\
\cline { 2 - 3 } & $13(43 \%)$ & $5(83 \%)$ & $>0.05$ (NS) \\
\hline $0.35 \mathrm{KU} / \mathrm{L}$ & $11(37 \%)$ & $1(17 \%)$ & \\
\hline
\end{tabular}

SPT: skin prick test; NS: non significant.

agrees with [8] who found that the most frequent clinical signs were cutaneous in $94 \%$ of cases with CMA. Three out of 30 patients $(10 \%)$ gave relevant symptoms suggestive of beef allergy, 2 gave urticarial symptoms and one gave gastrointestinal symptoms. This low percentage may be explained in part due to consumption of cooked beef which has a much lower allergenicity than raw and semi cooked [11].

\subsection{Results of Skin Prick Tests}

Six out of 30 patients (20\%) showed positive SPT results to beef. In Werfel study, a higher percentage was found in children (84\%) [6]. The processing methods for beef preparation used as SPT material were not reported in this study which can explain the discordance between results on basis of varied allergen concentrations and allergenicity in the test materials used [12].

\subsection{Results of Specific IgE}

In our study, 18 (60\%) out of 30 adults with CMA showed positive specific IgE results to beef, this represented IgE mediated beef allergy among IgE mediated adults with CMA. This was near with the results in children, where Mamikoglu (2005) conducted a study on 16 children who were allergic to milk, 14 of them (87.5\%) also were allergic to beef antigens according to their specific IgE results. [11].

We finally did the tests of agar gel precipitation for cross reactivity between milk and beef where the diameter of immuno-diffusion of milk was divided by that of beef. The percentage obtained was considered the cross reactivity between milk and beef for a particular patient. The mean percentage of all patients was $31.6 \% \pm 13.1 \%$.

\subsection{Overview}

From the above studies mentioned it is obvious that the prevalence of beef allergy in patients with CMA is difficult to infer from a review of the literature because samples are generally small and observations are heterogeneous. Furthermore, the diagnostic workup of beef allergy has not 
yet been standardized [3]. This may explain the discrepancy between the above studies together and therefore between them and our study.

Mamikoglu (2005) stated that patients who are allergic to milk may need to be tested for allergies to beef [11]; on the other hand Martorell et al. (2006) stated that beef does not need to be excluded from the diet of children allergic to CMA as long as it is eaten cooked [8]. This is because the allergen responsible for sensitization to beef being a bovine serum albumin that is thermo labile, which means that its allergic potential is destroyed by cooking [6] and it only produces a reaction when eaten raw or partially raw [12].

Martelli et al. (2002) agreed with such opinion as he stated that in patients with CMA, the low prevalence of beef allergy (13\% to $20 \%$ ) does not support the exclusion of beef from the diet except in selected cases [3]. Beef has high protein content, and nothing warrants its wholesale elimination from the diet of children who may have prolonged protein restrictions if also affected by multiple food allergies.

It was also noticed that most studies were conducted on pediatrics although our study found out that a great portion of adults suffer from the same problem. Another problem that there are no clear standards for such studies which are greatly needed to provide more accurate data.

Further long term studies should be done on this topic but, after setting standardizations of oral food challenge test doses. Additionally, more studies should be conducted on adults as most of the previous studies have been conducted on children.

\section{Conclusions}

Finally, according to our study, beef should not be eliminated from milk allergy patient's diet, except after investigations so as not to lose its nutritional value without benefit. Also, we can realize that negative skin test alone is insufficient to rule out milk and beef allergy and that specific IgE is more sensitive for diagnosis. A non IgE mediated allergy should be considered for cases with negative skin test and specific IgE. Further workup could be done using oral food challenge test or in vitro cross reactivity test.

\section{Acknowledgements}

The authors thank Dr. Selevia Tanago (MB, BCh) for coordination of clinical samples and our patients in Allergy and Clinical Immunology Department, Ain Shams University Hospital.

\section{REFERENCES}

[1] P. A. Eigenmann, “Anaphylaxis to Cow’s Milk and Beef
Meat Proteins," Annals of Allergy, Asthma and Immunology, Vol. 89, No. 6, 2002, pp. 61-64. doi:10.1016/S1081-1206(10)62125-4

[2] P. Restani, C. Ballabio, A. Cattaneo, P. Isoardi, L. Terracciano and A. Fiocchi, "Characterization of Bovine Serum Albumin Epitopes and Their Role in Allergic Reactions,” Allergy, Vol. 59, No. S78, 2004, pp. 21-24. doi:10.1111/j.1398-9995.2004.00568.x

[3] A. Martelli, A. De Chiara, M. Corvo, P. Restani and A. Fiocchi, " Beef Allergy in Children with Cow's Milk Allergy; Cow'S Milk Allergy in Children with Beef Allergy,” Annals of Allergy, Asthma and Immunology, Vol. 89, No. 6, 2002, pp. 38-43. doi:10.1016/S1081-1206(10)62121-7

[4] A. Paschke and M. Besler, "Stability of Bovine Allergens during Food Processing," Annals of Allergy, Asthma and Immunology, Vol. 89, No. 6, 2002, pp. 16-20. doi:10.1016/S1081-1206(10)62117-5

[5] H. Y. Lam, E. van Hoffen, A. Michelsen, K. Guikers, C. H. van der Tas, C. A. Bruijnzeel-Koomen and A. C. Knulst, "Cow’s Milk Allergy in Adults Is Rare but Severe: Both Casein and Whey Proteins Are Involved,” Clinical and Experimental Allergy, Vol. 36, No. 38, 2008, pp. 9951002. doi:10.1111/j.1365-2222.2008.02968.x

[6] S. J. Werfel, S. K. Cooke and H. A. Sampson, "Clinical Reactivity to Beef in Children Allergic to Cow's MilK," Journal of Allergy and Clinical Immunology, Vol. 99, No. 3,1997, pp. 293-300. doi:10.1016/S0091-6749(97)70045-9

[7] A. Fiocchi, P. Restani, E. Riva ,G. P. Mirri, I. Santini, L. Bernardo and C. L. Galli, "Heat Treatment Modifies the Allergenicity of Beef and Bovine Serum Albumin," Allergy, Vol. 53, No. 8, 1998, pp. 798-802. doi:10.1111/j.1398-9995.1998.tb03977.x

[8] A. Martorell, A. M. Plaza, J. Boné, S. Nevot, M. C. García Ara, L. Echeverria, E. Alonso, J. Garde, B. Vila, M. Alvaro, E. Tauler, V. Hernando and M. Fernández, "Cow’s Milk Protein Allergy. A Multi-Centre Study: Clinical and Epidemiological Aspects," Allergology and Immunopathology, Vol. 34, No. 2, 2006, pp. 46-53. doi:10.1157/13086746

[9] T. M. Li, T. Chuang, S. Tse, D. Hovanec-Burns and A. S. El Shami, "Development and Validation of a Third Generation Allergen-Specific IgE Assay on the Continuous Random Access IMMULITE 2000 Analyzer," Annals of Clinical and Laboratory Science, Vol. 34, No. 1, 2004, pp. 67-74.

[10] O. Outchterlony and L. A. Nilisson, "Immunodiffusion and immuno-Electrophoresis, Handbook of Experimental Immunology” In: D. M. Wier, Ed., Blackwell Scientific Publications, Oxford, 1986.

[11] B. Mamikoglu, "Beef, Pork, and Milk Allergy (Cross Reactivity with Each Other and Pet Allergies)," Otolaryngology, Head and Neck Surgery, Vol. 133, No. 4, 2005, pp. 534-537. doi:10.1016/j.otohns.2005.07.016

[12] J. D. Astwood, J. N. Leach and R. L. Fuchs, "Stability of Food Allergens to Digestion in Vitro," Natural Biotechnology, Vol. 14, No. 10, 1996, pp. 1269-1273. doi:10.1038/nbt1096-1269 